\title{
Enhancement of clavulanic acid production by mutant Streptomyces clavuligerus
}

\begin{abstract}
Background: Clavulanic acid (CA) is a potent $\beta$-lactamase inhibitor used to combat resistance to penicillin and cephalosporin antibiotics. There are several pathogenic microorganisms that are capable of secreting $\beta$-Lactamases. These enzymes catalyze the hydrolysis of the $\beta$-lactam ring of penicillins and cephalosporins, and their hydrolysis products have no antibiotic activity. Since the first clinical applications, the efficiency of the $\beta$-lactam antibiotics has been declining, due to the astonishing increasing number of bacteria capable of displaying $\beta$-lactam resistance. Thus, the genetic production improvement using physical and chemical mutagenic agents is an important strategy in programs of industrial production of bioactive metabolites, such as CA.
\end{abstract}

Objective: The objective of the study was to enhance CA production using chemical and UV mutagenesis on Streptomyces clavuligerus.

Methods: Streptomyces clavuligerus ATCC 27064 was used as the standard strain in all the experiments. The mutant strain was first obtained by chemical mutagenesis using the agent MMS and next the same strain undergoing one more mutation was obtained by UV light. Cellular biomass was analyzed based on the dry weight method and the concentration of CA was determined by HPLC analysis.

Results: CA production increased by $28 \%$ with mutant strain when compared to the wild-type strain. The mutant strain obtained $812 \mathrm{mg} \mathrm{L}-1$ concentration in $72 \mathrm{~h}$ of fermentation, while the wild-type strain obtained only $639.7 .1 \mathrm{mg} \mathrm{L}-1$ at the same time, and the productivity at this moment was 11.28 and $8.88 \mathrm{mg} \mathrm{L}-1 \mathrm{~h}-1$ respectively. The mutant strain had a significant increase in biomass growth between 36 and $60 \mathrm{~h}$.

Conclusion: The study showed that random mutations can lead to the improvement in CA production in $S$. clavuligerus. This finding reinforces the importance of using this method for productivity improvement.

Keywords: clavulanic acid, streptomyces clavuligerus, mutation, strain improvement
Volume 6 Issue 2 - 2018

\author{
Eliton da Silva Vasconcelos,' Raquel Fernanda \\ Salla ${ }^{2}$ \\ 'Department of Physiological Sciences, Federal University of São \\ Carlos, Brazil \\ ${ }^{2}$ Department of Animal Biology, State University of Campinas, \\ Brazil
}

Correspondence: Eliton da Silva Vasconcelos, Department of Physiological Sciences, Federal University of São Carlos, SP, Brazil,Tel +55 I63351 8968,Email elitonbio@hotmail.com

Received: January 13,2018 | Published: March 27, 2018
Abbreviations: ATCC, american type culture collection; CA, clavulanic acid; HPLC, high-performance liquid chromatography; MMS, methyl methanesulphonate; MOPS, 3-(N-Morpholino) propane sulfonic acid; UV, ultraviolet light

\section{Introduction}

$\beta$-lactam antibiotics are among the most popular classes of antibacterial agents, whose mechanism of action consists on the inhibition of bacterial cell wall synthesis. ${ }^{1}$ The production of $\beta$-lactamase is the most important mechanism for bacterial resistance to $\beta$-lactam antibiotics. ${ }^{2}$ Secondary metabolites produced by bacteria exhibit potent biological activities and have been developed extensively for antimicrobial, anticancer, and other vital therapeutic applications. ${ }^{3}$ In this sense, the CA is a secondary metabolite that was first detected in Streptomyces clavuligerus (S. clavuligerus) and is a potent inhibitor of a wide range of $\beta$-lactamases from pathogenic organisms. CA effectively inhibits the activity of $\beta$-lactamases of molecular classes A and D, such as cephalosporinases, penicillinases, and broad spectrum $\beta$-lactamases, and thus it has been widely used clinically to treat diseases caused by $\beta$-lactam resistant bacteria. ${ }^{4}$ The combined therapy of $\mathrm{CA}+\beta$-lactam antibiotic has been very successful in preventing infections due to Gram-positive (Staphylococcus sp.) and Gram-negative (Klebsiella sp. Hemophilus sp. Proteus, Shigella, Pseudomonas) $\beta$-lactamase producing pathogens. ${ }^{1}$

Chemical mutagenesis and UV light based random mutagenesis are some of the approaches to isolate interesting mutations with the potential of single nucleotide changes. Chemical mutagenesis can be performed using a variety of chemicals that cause DNA damage, leading to single nucleotide changes and/or deletions, while UV light leads to DNA damage both directly and through the generation of reactive oxygen species. ${ }^{5}$ Most Streptomyces species studied are unstable for certain characters (i.e., a spontaneous mutation rate higher than $0.1 \%$ per spore). The chromosome is very unstable and undergoes very large deletions spontaneously. This genetic instability can be stimulated by mutagens, such as mitomycin $\mathrm{C}$, ultraviolet (UV) light and treatments with chemical agents. ${ }^{6,7} \mathrm{UV}$ irradiation is one of the strain improvement strategies through random mutation. UV is a very convenient and relatively safe method, and in the range of $200-300 \mathrm{~nm}$ it produces thymidine dimmers and increases the probability of deletion during the duplication process. ${ }^{8} \mathrm{UV}$ irradiation can also increase the concentration of CA up to two-fold, ${ }^{5,9}$ as it also makes their production cheaper. These molecular changes affect different phenotypical properties, often pleiotropically, including morphological differentiation, production of secondary metabolites 
(pigments and antibiotics), antibiotic resistance, secretion of extracellular enzymes and sometimes genes for primary metabolism, particularly one or more steps in the arginine biosynthetic pathway. ${ }^{7}$

The increased resistance to the currently recommended antibiotics is responsible for several human deaths and it has been a constant concern. In addition, the major cause of antibiotic resistance is the inappropriate use and excessive treatment of antibiotics. Thus, the improvement of microbial strains plays an important role to improve antibiotic production, reducing the production costs during industrial fermentation. Random mutagenesis and screening approaches are relevant methods to enhance strain productivity by eliminating the competing pathways and the structurally similar molecules, which leads to a more efficient overall process. These methods eliminate unrelated secondary metabolic pathways from the strain and make general cellular resources available for the specific pathway of interest, which could be explored further as a general approach to improve strain productivity. ${ }^{1}$

In this context, this study reveals a relevant amount of CA production by mutant strain obtained from $S$. clavuligerus. To improve the production of $\mathrm{CA}$, the following three main phases were considered: (i) treatment with chemical agent MMS for strain improvement; (ii) a second treatment using UV for strain improvement; (iii) selection of a culture medium for mutant strain reported in previous studies. ${ }^{10-12}$

\section{Microorganism strains}

Streptomyces clavuligerus ATCC 27064 (www.atcc.org) was used as the standard strain in all the experiments. This strain underwent two mutations in order to improve the production of CA. The mutant strain was first obtained by chemical mutagenesis using the agent MMS following the technique described by Stonesifer \& Baltz. ${ }^{13}$ The mutant strain, when cultivated in solid medium, lacked pigmentation in their mature spores (data not shown), being classified as whi mutants ("white" - Chater ${ }^{14}$ ). After MMS mutation, the same strain undergoing one more mutation was obtained by UV light, as described by Lee et al. ${ }^{8}$ The wild-type strain is classified in the gray series of category IV from Streptomyces genera, based on the dark greenish pigmentation shown by its mature spores..$^{15}$ All of the microorganisms were conserved as vegetative cell suspensions in cryoprotective $10 \%$ $\mathrm{p} / \mathrm{v}$ glycerol stocks stored at $-70^{\circ} \mathrm{C}$.

\section{Culture media}

The wild-type and the mutant strains were initially cultivated in reactivation medium (composition in g.L-1 in distilled water), glycerol, 15.0; bacto-peptone, 10.0; malt extract 10.0; yeast extract 1.0; $\mathrm{K}_{2} \mathrm{HPO}_{4}, 2.5 ; \mathrm{MgSO}_{4} .7 \mathrm{H}_{2} \mathrm{O}, 0.75 ; \mathrm{MnCl}_{2} .4 \mathrm{H}_{2} \mathrm{O}, 0.001 ; \mathrm{FeSO}_{4} .7 \mathrm{H}_{2} \mathrm{O}$, 0.001 ; $\mathrm{ZnSO}_{4} .7 \mathrm{H}_{2} \mathrm{O}, 0.001$; in MOPS buffer, $21.0(100 \mathrm{mM})$. The medium $\mathrm{pH}$ was adjusted to $\mathrm{pH} 6.8$ with titration of $\mathrm{NaOH} 5 \mathrm{M}$ solution and then sterilized. Reactivated cells were then inoculated in the respective mediums used in the CA production assays.

In the next phase, the semi synthetic medium GSPA was used. ${ }^{10}$ GSPA medium composition was (g.L-1 in distilled water): glycerol, 15.0; sucrose, 20.0; proline, 2.5; glutamic acid 1.5; arginine, 10.0; $\mathrm{NaCL}, 5.0 ; \mathrm{K}_{2} \mathrm{HPO}_{4}, 2.0 ; \mathrm{CaCl}_{2}, 0.4 ; \mathrm{MnCl}_{2} .4 \mathrm{H}_{2} \mathrm{O}, 1.0 ; \mathrm{FeCl}_{3} .6 \mathrm{H}_{2} \mathrm{O}$, $0.1 ; \mathrm{ZnCl}_{2}, 0.05 ; \mathrm{MgSO}_{4} .7 \mathrm{H}_{2} \mathrm{O}, 1.0 ; \mathrm{pH} 7.0$. This specific medium was used due to good results in our laboratory investigating production of CA with mutant strains in a previous study. ${ }^{12}$

\section{Culture conditions}

Batch cultivations for the $S$. clavuligerus CA production were done in a shaker G-25 (New Brunswick Scientific Co). The assays were done in three steps, strain reactivation, microorganism growth and CA production. In the reactivation step, $3.5 \mathrm{~mL}$ of the $S$. clavuligerus vegetative cell suspension stock was inoculated in $500 \mathrm{~mL}$ Erlenmeyers containing $50 \mathrm{~mL}$ of reactivation medium, and incubated for $24 \mathrm{~h}$ at $28^{\circ} \mathrm{C}, 250 \mathrm{rpm}$.

In the growth step, $5 \mathrm{~mL}$ of the reactivated $S$. clavuligerus suspension was transferred to $500 \mathrm{~mL}$ Erlenmeyers containing $45 \mathrm{~mL}$ of culture mediums, each one with the same composition relative to the main CA production mediums. The growth step was carried over $24 \mathrm{~h}$ at $28^{\circ} \mathrm{C}, 250 \mathrm{pm}$. Finally, in the production step, the whole growth culture volumes were transferred into Erlenmeyers containing the production mediums, beginning the main CA production process. CA production cultures were carried over $120 \mathrm{~h}$ and the samples from the cultures were collected every $12 \mathrm{~h}$.

\section{Biomass and clavulanic acid determination}

Cellular biomass was analyzed based on the dry weight method. First, the cells were collected by centrifugation of the samples, washing the cells twice with double distilled water. The samples were then incubated at $65^{\circ} \mathrm{C}$ until reaching the measured constant weight. CA concentration was determined by HPLC using the Foulstone \& Reading ${ }^{16}$ method, in a C- $18 \mu$-Bondapack column. The mobile phase was a methanol/phosphate buffer mix, at $2.5 \mathrm{~mL} / \mathrm{min}-1$ flow rate. Elution temperature was kept at $28^{\circ} \mathrm{C}$ and was monitored at $311 \mathrm{~nm}$ wavelength. A reference calibration curve was previously done using an amoxicillin/potassium clavulanate mix (the content of Clavulin 250mg - Smith Kline-Beecham do Brasil Ltda).

\section{Statistical analysis}

All the analyses and experiments were performed in triplicate. Data are shown as means \pm SEM. Differences between the WT and Mutant groups were compared by the unpaired t-test. To compare changes in each variable over time one-way ANOVA was performed followed by the Tukey and Kramer post-hoc test. The differences were considered significant at $\mathrm{P}<0.05$. Data were analyzed using GraphPad InStat 3.00 software (GraphPad Software, San Diego, CA, USA).

\section{Results and discussion}

CA is an industrially important secondary metabolite due to its inhibitory action on b-lactamases. There are many examples of strain improvement strategies applied to S. clavuligerus in order to reach high levels of CA production with genetic engineering and random mutagenesis. ${ }^{9,17}$

In this study, we used random mutagenesis and our results showed that CA production increased $28 \%$ within the mutant strain when compared to the wild-type strain. The level of CA reached a maximum after $72 \mathrm{~h}$ of cultivation in mutant and wild-type strains. The mutant strain obtained $812 \mathrm{mg} \mathrm{L}-1$ concentration in $72 \mathrm{~h}$ of fermentation, while the wild-type obtained $639.7 .1 \mathrm{mg} \mathrm{L}-1$ at the same time (Figure 1). This concentration of CA in the mutant strain was similar at our previous study, ${ }^{12}$ in which only the chemical mutagenesis was used. 


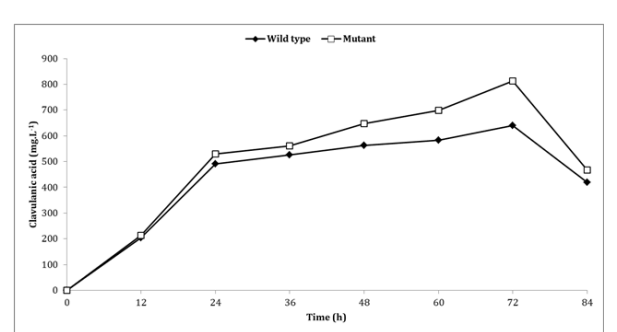

Figure I Comparison of clavulanic acid production between the mutant and wild-type strains cultivated in semi-synthetic mediums GSPA.

The significant increase in biomass in the mutant strain compared to wild type (Figure 2), suggests that the mutations occurred in the genes which are involved in the primary metabolites responsible for growth. It might be interpreted that the overproduction of CA, following the mutations, is a result of overgrowth. We also suggest that mutations in the genes involved in the metabolic pathway of CA production may have eliminated the competing pathways of structurally similar molecules, leading to a more efficient CA production.

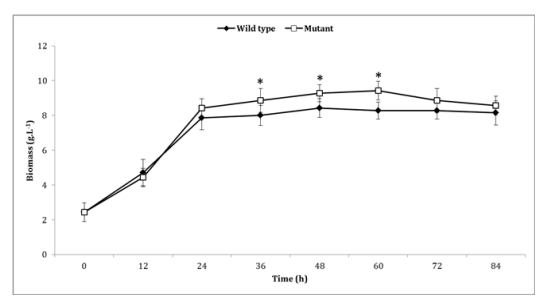

Figure 2 Comparison of biomass between the mutant and wild-type strains cultivated in semi-synthetic mediums GSPA. Mean values \pm SEM.Asterisks indicate significant differences between wild-type and mutant strains $(\mathrm{P}<$ 0.05).

According to Medema et al., ${ }^{18}$ random mutagenesis can cause gene transcript changes in both primary and secondary metabolism. Some random mutagenesis were strikingly similar to those rationally engineered by two strategies that have recently been employed to increase CA production in S. clavuligerus: redirection of carbon fluxes towards the key CA precursor glyceraldehyde-3-phosphate (G3P), and upregulation of pathway-specific activators. ${ }^{18}$ In this study, the highest productivity of the two strains occurred in $72 \mathrm{~h}$ of cultivation. The productivity of the wild-type and the mutant strains were 8.88 and $11.28 \mathrm{mg} \mathrm{L}^{-1} \mathrm{~h}^{-1}$ respectively (Table 1 ).

Table I Maximum clavulanic acid concentration and productivity of wildtype and mutant strains

\begin{tabular}{llll}
\hline Strain & $\begin{array}{l}\text { Maximum CA } \\
\text { concentration }(\mathbf{m g} \\
\left.\mathbf{L}^{-1}\right)\end{array}$ & $\begin{array}{l}\text { Production } \\
\text { time }(\mathbf{h})\end{array}$ & $\begin{array}{l}\text { Productivity } \\
\left(\mathbf{m g ~ L}^{-1} \mathbf{h}^{-1}\right)\end{array}$ \\
\hline Wild type & 639.72 & 72 & 8.88 \\
Mutant & 812.71 & 72 & 11.28 \\
\hline
\end{tabular}

These results show that although laborious and empirical, the strategy of random mutation and screening can be a good method for improvement in CA production. The improvement of microbial strains plays an important role in reducing production costs during industrial fermentation. Korbekandi et al. ${ }^{9}$ reported that the concentration of CA was increased 1.8 times after UV mutagenesis. In another study,
Said et al. ${ }^{19}$ show that relatively safe mutagens like UV represent a very convenient system to enhance strains through random mutation. On the other hand, only a small number of researchers have utilized UV irradiation to overproduce CA. According to Baltz, ${ }^{20}$ chemical mutagenesis is a very successful method to improve secondary metabolites in Streptomyces. It is important to emphasize that the GSPA culture medium was selected because this medium has high concentration of arginine. Indeed the primary metabolic precursors of CA are D-glyceraldehyde-3-phosphate $(\mathrm{G} 3 \mathrm{P})^{21}$ and L-arginine. ${ }^{22}$ Li \& Townsend ${ }^{11}$ have already shown that the addition of arginine to the cultured mutant strain further improved CA production giving a greater than two-fold increase over the wild-type. In addition, the authors show that this amino acid is fundamental for CA biosynthesis and your lack is a limiting factor.

This study showed that random mutagenesis like chemical and UV irradiation are methods safe and can be used for improvement $\mathrm{AC}$ production as well as other biosynthesis metabolites, altering genes and consequently levels of these substances. More guidance acts are necessary to inappropriate use and excessive treatment of antibiotics, thereby avoiding several human deaths. Additionally, a better understanding of the mechanisms involved in the production of $\mathrm{CA}$ as well as studies aimed at increasing production it is necessary.

\section{Conclusion}

Our findings clearly demonstrate that random mutations can lead to the improvement in CA production in S. clavuligerus. The highest levels of CA production obtained within the mutant strain reached $28 \%$ more than the levels of the wild-type strain. However, adding another mutation in the same mutant strain, in this case, UV light does not improve the production. The semi synthetic medium GSPA promoted an excellent biomass growth and CA production with both strains. This work reinforces the importance of using random mutagenesis methods in $S$. clavuligerus for biotechnological research of productivity improvement.

\section{Acknowledgements}

None.

\section{Conflict of interest}

The authors declare there is no conflicts of interest.

\section{References}

1. Paradkar A. Clavulanic acid production by Streptomyces clavuligerus: biogenesis, regulation and strain improvement. J Antibiot. 2013;66(7):110 .

2. Bush K. Characterization of beta-lactamases. Antimicrob Agents Chemother. 1989;33(3):259-263.

3. Craney A, Ozimok C, Pimentel-Elardo SM, et al. Chemical perturbation of secondary metabolism demonstrates important links to primary metabolism. Chem Biol. 2012;19(8):1020-1027.

4. Qin R, Zhong C, Zong G, et al. (2017) Improvement of clavulanic acid production in Streptomyces clavuligerus F613-1 by using a claR-neo reporter strategy. Electron J Biotechnol. 28:41-46.

5. Bose JL. Chemical and UV Mutagenesis. Methods Mol Biol. 2016;1373:111-115. 
6. Dharmalingam K, Cullum J. Genetic instability in Streptomyces. J Biosci. 1996;21(3):433-444.

7. Volff JN, Vandewiele D, Simonet JM, et al. Ultraviolet light, mitomycin C and nitrous acid induce genetic instability in Streptomyces ambofaciens ATCC23877. Mutat Res. 1993;287(2):141-156.

8. Lee SD, Park SW, Oh KK, et al. Improvement for the production of clavulanic acid by mutant Streptomyces clavuligerus. Lett Appl Microbiol. 2002;34(5):370-375.

9. Korbekandi H, Darkhal P, Hojati Z, et al. Overproduction of Clavulanic Acid by UV Mutagenesis of Streptomyces clavuligerus. Iran J Pharm Res. 2010;9(2):177-181

10. Romero J, Liras P, Martin JF. Utilization of ornithine and arginine as specific precursors of clavulanic acid. Appl Environ Microbiol. 1986;52(4):892-897.

11. Li R, Townsend CA. Rational strain improvement for enhanced clavulanic acid production by genetic engineering of the glycolytic pathway in Streptomyces clavuligerus. Metab Eng. 2006;8(3):240-352.

12. Vasconcelos ES, Lima VA, Goto LS, et al. Clavulanic acid production by the MMS 150 mutant obtained from wild-type Streptomyces clavuligerus ATCC 27064. Braz J Microbiol. 2013;44(4):1049-1057.

13. Stonesifer J, Baltz R. Mutagenic DNA repair in Streptomyces. Proc Nat Acad Sci USA. 1985;82(4):1180-1183.

14. Chater KF. Regulation of sporulation in Streptomyces coelicolor A3(2): a checkpoint multiplex? Curr Opin Microbiol. 2001;4(6):667-673.
15. Locci R. Streptomyces and related Genera. Bergey's Manual of Systematic Bacteriology. In: Williams ST, Sharpe MD, Holt JG,1 editors. Williams \& Wilkins, Baltimore, USA; 1989:2451-2492.

16. Foulstone M, Reading C. Assay of amoxicillin and clavulanic acid, components of augmentin, in biological fluids with performance liquid chromatography. Antimicrob Agents Chemother. 1982;22(5):753-762.

17. Kizildogan AK, Jaccard GV, Mutlu A, et al. Genetic engineering of an industrial strain of Streptomyces clavuligerus for further enhancement of clavulanic acid production. Turkish J Biol. 2017;41:342-353.

18. Medema MH, Alam MT, Heijne WH, et al. Genome-wide gene expression changes in an industrial clavulanic acid overproduction strain of Streptomyces clavuligerus. Microb Biotechnol. 2011;4(2):300-305.

19. Said IG, Abdelwahed NAM, Awad HM, et al. Enhancement of clavulanic acid production by Streptomyces sp MU-NRC77 via mutation and medium optimization. Trop J Pharm Res. 2017;16(1):31-42.

20. Baltz RH. New genetic methods to improve secondary metabolite production in Streptomyces. J Ind Microbiol Biotechnol. 1998;20(6):360 363.

21. Khaleeli N, Li RF, Townsend CA. Origin of the b-lactam carbons in clavulanic acid from an unusual thiamine pyrophosphate mediated reaction. J Am Chem Soc. 1999;121(39):9223-9224.

22. Valentine BP, Bailey CR, Doherty A, et al. Evidence that arginine is a later metabolic intermediate than ornithine in the biosynthesis of clavulanic acid by Streptomyces clavuligerus. J Chem Soc Chem Commun. 1993;15:1210-1211. 